Published in final edited form as:

Nat Methods. 2014 November ; 11(11): 1131-1134. doi:10.1038/nmeth.3139.

\title{
Time-resolved crystallography using the Hadamard Transform
}

\author{
Briony A. Yorke ${ }^{1,4}$, Godfrey S. Beddard ${ }^{2,5}$, Robin L. Owen ${ }^{3}$, and Arwen R. Pearson ${ }^{1,4}$ \\ ${ }^{1}$ Astbury Centre for Structural Molecular Biology. The University of Leeds, Leeds, LS2 9JT, UK \\ ${ }^{2}$ School of Chemistry. The University of Leeds, Leeds, LS2 9JT, UK \\ ${ }^{3}$ Diamond Light Source, Harwell Science and Innovation Campus, Didcot, UK
}

\begin{abstract}
A new method for performing time-resolved X-ray crystallographic experiments based on the Hadamard Transform is proposed and demonstrated. The time-resolution is defined by the underlying periodicity of the probe pulse sequence and the signal to noise is greatly improved compared to the fastest experiments depending on a single pulse. This approach is general and equally applicable to any spectroscopic or imaging measurement where the probe can be encoded.
\end{abstract}

\section{Introduction}

The ability to watch chemistry and biology happen has huge potential to add to our understanding of molecular function in many contexts. There is increasing interest in targeting specific protein conformational states as a route to higher drug specificity and selectivity ${ }^{1}$ and functional materials that respond to changes in their environment represent new possibilities for sensors, energy and information storage ${ }^{2,3}$. All these research areas require an understanding of both structure and dynamics. There are numerous examples of high-resolution crystal structures of reactive molecules and macromolecules, providing static models from which to infer function. In many cases, however, the reaction mechanisms remain imperfectly understood, usually because intermediate species are too short-lived to observe by conventional diffraction methods. In contrast, spectroscopic methods enable transient molecular species with lifetimes as short as femtoseconds to be observed $^{4}$ but do not usually provide structural information, leaving the challenge of relating structure to function unresolved. Time-resolved X-ray crystallography (TRX) has begun to

\footnotetext{
Users may view, print, copy, and download text and data-mine the content in such documents, for the purposes of academic research, subject always to the full Conditions of use:http://www.nature.com/authors/editorial_policies/license.html\#terms

Correspondence to: arwen.ruth.pearson@uni-hamburg.de, g.s.beddard@leeds.ac.uk, robin.owen@diamond.ac.uk.

${ }^{4}$ Current Address: Hamburg Centre for Ultrafast Imaging, Universität Hamburg, Hamburg, Germany

${ }^{5}$ Current Address: School of Chemistry, University of Edinburgh, Edinburgh, Scotland, U.K.

Author Contributions

GSB and BAY originally proposed the application of the Hadamard Transform to time-resolved experiments. BAY, RLO and ARP devised the proof-of-principle crystallographic experiment and collected the data. BAY, RLO, GSB and ARP all processed data, wrote software and jointly wrote the manuscript.

Accession Codes

The low-dose thaumatin structure refined against HATRX summed data time-point 1 is available from the PDB (www.pdb.org) under the accession codes: $4 \mathrm{C} 3 \mathrm{C}$ (coordinates) \& R4C3CSF (structure factors).

There are no competing financial interests.
} 
meet these challenges ${ }^{5,6}$, but so far has only provided high resolution structural information for short-lived, i.e. sub millisecond, intermediates of a small number of proteins.

Although ultra-fast methods for time-resolved spectroscopy are well established, this is not the case for crystallography. The current state-of-the-art methods for TRX are the Laue $\operatorname{method}^{7,8}(\sim 100 \mathrm{ps})$ and streak camera techniques or bunch slicing methods where $\sim 1$ ps or better time resolution has been obtained for molecular samples ${ }^{9,10}$. Such high timeresolution can be achieved because there are sufficient photons in each single X-ray probe pulse that measurable diffraction data can be obtained. Recent developments in X-ray free electron lasers offer the possibility of femtosecond TRX ${ }^{11}$. Such high time-resolutions cannot be achieved at a conventional monochromatic beamline for a macromolecular sample because there are simply not enough X-ray photons in a period of less than $1 \mu$ s to obtain a useable diffraction pattern ${ }^{12}$.

The conventional way to study transient phenomena in spectroscopic or crystallographic experiments is to initiate the process and then to probe the system at a later time, either with $\mathrm{X}$-rays ${ }^{13}$ or visible/infrared (IR) photons ${ }^{4,14,15}$. In classical time-resolved experiments a laser (pump) pulse is used for initiation and a single probe pulse follows every pump pulse at a series of predetermined time delays. To measure $n$ time points $n$ pump-probe pairs are needed (Fig. 1a).

The Hadamard time-resolved approach presented here is a transform method. These are common in spectroscopy but have so far not been used in time-resolved measurements. In contrast to the conventional pump-probe method, in our Hadamard approach each pump pulse is followed by a sequence of probe pulses and the total signal from each sequence is recorded in a single measurement (Fig. 1b). The sensitivity of the experiment is therefore defined by the number of photons within the entire probe sequence, with the time resolution defined as the total probe sequence length divided by the number of pulses.

As in classical pump-probe, $n$ pump-probe sequences are needed to measure $n$ time points. The pattern of the probe sequence can be represented as rows of a $n \times n$ matrix (S) derived from a Hadamard sequence (Supplementary Fig. 1, Supplementary Note 1) ${ }^{16}$. As shown for the simplest case of three time points (Fig. 1b), each row of the matrix (and hence probe sequence) is obtained by a cyclic left shift by one element from the previous row (Fig. 1c).

For a Hadamard time-resolved experiment the reaction is initiated, and then the entire probe sequence (given by the first row of the $\mathbf{S}$ matrix) is recorded by the detector as a single image. This is repeated on a new sample (or on the same sample after relaxation), but with the probe sequence now defined by the next row of the $\mathbf{S}$ matrix, until all rows have been used. The resulting encoded signals, from $n$ excitations are collated to form a vector $\boldsymbol{W}$ of length $n$. To obtain the time-dependent signal, $I_{t}$, the probe sequence encoding is reversed by multiplying the vector $\boldsymbol{W}$ by the inverse of the matrix $\mathbf{S}$. i.e. $I_{t}=\mathbf{S}^{-1} \boldsymbol{W}$.

To our knowledge, Hadamard encoding has not been previously applied to time-resolved experiments but has been used to improve the signal to noise ratio in optical and IR imaging and spectroscopy ${ }^{16}$, mass spectrometry ${ }^{17}$ and NMR spectroscopy ${ }^{18}$. Excitingly, the use of Hadamard sequences for time-resolved measurements is generally applicable to any time- 
resolved experiment where the probe beam can be encoded, such as transient absorption spectroscopy. Here we demonstrate the validity of this approach by observing X-ray induced, time dependent changes in thaumatin crystals ${ }^{19}$ in a Hadamard time-resolved crystallographic (HATRX) experiment.

\section{Experimental details \& results}

The use of the Hadamard approach is independent of the manner in which the pulse sequence is generated. A detector encoded HATRX experiment can be reproduced by selectively summing single images from a continuous series of evenly spaced exposures into HATRX images. This allows comparison of the HATRX electron density to that from a control time-series acquired in the conventional manner, to demonstrate that a HATRX experiment can successfully track time-dependent structural changes (Fig. 2).

Exposure of protein crystals to the microfocus X-ray beamline I24 (Diamond Light Source) results in fast, irreversible radiation damage that can be readily observed through progressive breakage of disulphide bonds ${ }^{19}$. In this proof-of-principle experiment, the absorbed X-ray dose was used as a proxy for time with the X-ray beam itself acting as the "pump". A $3 \times 3 \mathbf{S}$ matrix was used, but to extend the dose (time) range probed this was repeated 9 times to give a total of 27 time-points. Diffraction datasets (wedges) consisting of 27 repeated images of the same $1^{\circ}$ oscillation were collected. Equivalent wedges of data were also recorded from an independent set of crystals to provide a control dataset with known intensities at each time point. The exposure time and incident flux were such that significant radiation damage accumulated within each wedge with the experimental dose limit reached by the final image ${ }^{20}$.

For the control data, composite scaled datasets for each time point were created (Fig. 3a, Supplementary Table 1). To create the HATRX datasets, wedges were assigned an exposure sequence corresponding to a single line of the $n=3 \mathbf{S}$ matrix (Fig. 3b) and summed into nine HATRX datasets encoding all 27 time-points. Both the HATRX and the control images were integrated and scaled using standard methodology (Online Methods).

Each $3 \times 3$ HATRX experiment is comprised of 3 datasets, each encoded with one line of the $\mathbf{S}$ matrix (Supplementary Table 2). For each dataset, the intensities of all reflections were converted into amplitudes and then collated into a vector $\boldsymbol{W}$. This was transformed to yield 3 datasets now corresponding to 3 time (dose) points (Supplementary Software). This was done for all 9 HATRX experiments. The resulting structure factors for the control and HATRX data were the same within experimental error (Supplementary Table 3). The same was true for data treated as a 7-period HATRX experiment, now repeated 4 times, although in this case the data completeness was insufficient for map calculation $(<60 \%)$.

$F_{\text {HATRX }}-F_{\text {CONTROL }}$ electron density maps were calculated for each time point and these showed no significant difference features at 3 r.m.s.d. However, $F_{\mathrm{t}=\mathrm{n}}-F_{\mathrm{t}=0}$ electron density maps for both the HATRX and control data show similar clear significant electron density changes at the disulphide bonds, as expected, with increasing X-ray dose (Fig. 2, 
Supplementary Fig. 3). These results demonstrate that the HATRX method produces reliable 4D electron density maps, which are simultaneously highly resolved in both space and time.

\section{Discussion}

We have demonstrated a new and generally applicable transform method for performing time-resolved experiments. The HATRX method frees the achievable time resolution from its current dependence on source brilliance by summing time-points across a probe sequence. This results in an improved signal to noise ratio ${ }^{16}$, because of the increased number of photons recorded during each measurement.

A comparison of the expected signal can be made using estimates of the known flux from a monochromatic X-ray beamline. For example, beamline I24 delivers $\approx 10^{12}$ photons s$^{-1}$. Assuming an elastic scattering efficiency of $0.1 \%,{ }^{13}$ for an image with 100 spots there are $10^{7} \mathrm{photons} \mathrm{scattered/spot/s} \mathrm{(assuming} \mathrm{for} \mathrm{illustration} \mathrm{that} \mathrm{spots} \mathrm{are} \mathrm{of} \mathrm{equal} \mathrm{intensity).} \mathrm{If}$ we record $1001 \mu$ s time points over a $100 \mu$ s range then with classical pump-probe each spot will contain only 10 photons. In contrast, by using the HATRX approach each spot will contain approximately 500 photons, because the HATRX sequence is approximately half on and half off (Supplementary Fig. 2).

There are two contributions to the improvement in signal to noise. The first is the absolute number of photons recorded for each time point. Consider the simplest $n=3$ experiment. In the classical pump-probe approach, each time-dependent measurement is made once.

However, with the HATRX approach, the encoded measurement is repeated 3 times with each time point measured twice, resulting in a doubling of the signal recorded.

Consequently, the mean square error associated with the measurement is reduced by a factor $(n+1)^{2} / 4 n \approx n / 4$ so the signal-to-noise ratio is increased by a factor of $(n+1) / 2 \sqrt{n}_{n} \approx \sqrt{n}_{n}$ / 2 relative to conventional experiments ${ }^{16}$ (Supplementary Note 1 ).

Although we have used a sequence of probe pulses that are equally separated in time, the method will also work if the probe pulses are logarithmically spaced, allowing a wide range of time-scales to be observed in a single experiment. The experiment measures the total intensity from a sequence and is independent of the time-stamp of each pulse.

An additional advantage of HATRX, specific to crystallography, is that the scaling of data from multiple crystals and time-points need only be done once (as the same sample population progresses in time). In contrast, in a serial crystallography pump-probe experiment the data must be scaled first for each time-point over all crystal orientations and then again over time.

Experimentally, generating Hadamard pulse sequences for HATRX could be achieved in a number of ways; using a rotating disc shutter encoded with a sequence or by deflecting electron bunches out of the synchrotron electron beam. An alternative approach is to use a pixel array detector gated to record according to the Hadamard pulse sequence but reading out only after the sequence is completed (Supplementary Note 2). 
HATRX thus enables fast TRX experiments on both reversible and irreversible processes at synchrotron sources where currently the flux density achievable at the sample is the limiting factor. Though HATRX is demonstrated here with millisecond time-resolution, it can be extended to shorter time-scales (Supplementary Note 2). This removes a significant experimental barrier and greatly increases the number of synchrotron beamlines where TRX experiments are possible. In addition, its applicability to any experimental technique where the probe can be encoded makes this a general tool for dynamic studies.

\section{Online Methods}

\section{Crystallization and data collection}

Thaumatin is a protein that contains eight disulphide bonds and is well characterised in radiation damage studies ${ }^{19}$. Thaumatin crystals were prepared as follows. The protein in $\mathrm{ddH}_{2} 0[40 \mathrm{mg} / \mathrm{ml}]$ was crystallised in sitting drop plates in a 2:1 mixture (4 $\mu \mathrm{l}$ protein: $2 \mu \mathrm{l}$ reservoir solution) with $0.05 \mathrm{M} \mathrm{ADA} \mathrm{pH} 6.8,0.6 \mathrm{M} \mathrm{K} / \mathrm{Na}$ Tartrate and 20\% v/v glycerol. The crystals ( 10-20 $\mu \mathrm{m}$ along the longest edge) were then mounted on a polyimide mesh (MiTeGen) and cryo-cooled to $100 \mathrm{~K}$ in liquid nitrogen. All diffraction data were collected on beamline I24 at the Diamond Light Source (Didcot, UK) at $100 \mathrm{~K}$, with a beam size of 10 $\times 10 \mu \mathrm{m}^{2}$ and an incident flux of $1.5 \times 10^{12} \mathrm{ph} \mathrm{s}^{-1}$. Wedges of data were collected from 264 microcrystals. These wedges contained 27 images taken over a repeated oscillation of $1^{\circ}$, each image had an exposure time of $200 \mathrm{~ms}$. The absorbed dose was calculated using RADDOSE $^{21}$ and the total absorbed dose was 29 MGy over the 27 images.

\section{Generation \& processing of Hadamard Series data}

A subset of 31 crystals was used for the control dataset. The diffraction data were integrated using $\mathrm{XDS}^{22}$ and then scaled together (but not merged) into a single .mtz file where the image numbers associated with each reflection were retained. The data from all image 1's were then scaled together using AIMLESS ${ }^{23}$ to produce a dataset for time (dose) point 1. This was repeated for each time point to yield 27 time-resolved datasets. The merging statistics for the resulting datasets are shown in Supplementary Table 1.

The data wedges from the remaining 233 crystals were split into three approximately equal groups. An exposure sequence corresponding to a line of the $3 \times 3 \mathbf{S}$ matrix was assigned to each group to determine which images would be summed for the HATRX analysis. The required images were then summed using the programme SUMSUB provided by David Waterman (CCP4) to yield a new HATRX encoded image that could then be indexed and integrated as normal (Fig. 3b). Scripts used for the creation of the HATRX data can be found in Supplementary Software.

The resulting HATRX images were indexed and integrated using XDS and then scaled using AIMLESS. Some poorly merging wedges from each group were discarded resulting in 72, 76 and 70 wedges finally used for each HATRX group (Supplementary Table 2). The intensities were converted to amplitudes using TRUNCATE ${ }^{24}$ and the $F$ and $S i g F$ for each reflection were collated into the vector $\boldsymbol{W}$ for each of the 9 HATRX datasets.

Multiplication of these vectors (for both $F$ and $\operatorname{Sig} F$ ) by the inverse of the $\mathbf{S}$ matrix, 


$$
\left[\begin{array}{c}
I_{1} \\
\vdots \\
I_{n}
\end{array}\right]=\mathbf{S}^{-1}\left[\begin{array}{c}
W_{1} \\
\vdots \\
W_{n}
\end{array}\right]
$$

yielded 3 datasets corresponding to 3 time (dose) points. This was repeated for the next eight sets i.e. batch groupings of $(4,5,6),(7,8,9)$ etc. to yield 27 time-resolved datasets (Supplementary Fig. 4). Scripts used for the HATRX transformation can be found in Supplementary Software.

Within the error of the experiment the control and HATRX data were indistinguishable (Supplementary Table 3). The same was true for data treated as for a 7-period HATRX experiment ( 7 groups of 25 crystals used, i.e. batch groupings of (1-7), (8-14) etc. to yield 4 HATRX images, data not shown), although in this case the data completeness was insufficient to allow for map calculation $(<60 \%)$.

Phases were calculated using $1 \mathrm{KWN}$ as a starting model. The model was refined using REFMAC $5^{25}$ against the HATRX time-point 1 data and the resulting phases used for all further map calculations (Supplementary Table 5). Maps derived from the control and HATRX data were then compared by the calculation of difference maps.

i.e. $F_{\mathrm{HATRX}_{1}}-F_{\mathrm{TRAD}_{1}}$ for $1 \leq n \geq N$; $\left(F_{\mathrm{HATRX}_{\mathrm{N}}}-F_{\mathrm{HATRX}_{1}}\right) v$ s. $\left(F_{\mathrm{TRAD}_{\mathrm{N}}}-F_{\mathrm{TRAD}_{1}}\right)$.

\section{Supplementary Material}

Refer to Web version on PubMed Central for supplementary material.

\section{Acknowledgments}

BAY is supported by the Wellcome Trust 4 year PhD programme "The Molecular Basis of Biological Mechanisms" 089312/Z/09/Z. This work was also supported by the EPSRC Award; "Dynamic Structural Science at the Research Complex at Harwell" EP/I01974X/1 and BBSRC Award BB/H001905/1. Diffraction data were collected on beamline I24 at Diamond Light Source under proposal nt5810. We thank Diamond Light Source and the staff of I24 for beamtime and support and D. Waterman (CCP4) for the SUMSUB code. We also thank P. Raithby, C. Wilson, J. Trincao, J. Christensen, M. Hamilton, E. Snell, T. Burnley, C. Wood and C. Ballard for useful discussions and critical reading of the manuscript.

\section{References}

1. Copeland RA. Future Med. Chem. 2011; 3:1491-1501. [PubMed: 21882942]

2. Giuseppone N, Fuks G, Lehn J-M. Chemistry. 2006; 12:1723-1735. [PubMed: 16392112]

3. Buryak A, Severin K. Angew. Chem. Int. Ed. Engl. 2005; 44:7935-7938. [PubMed: 16281316]

4. Zewail, AH. Femtochemistry: Ultrafast Dynamics of the Chemical Bond I. World Scientific; 1994.

5. Bourgeois D, Weik M. Crystallogr. Rev. 2009; 15:87-118.

6. Neutze R, Moffat K. Curr. Opin. Struct. Biol. 2012; 22:651-659. [PubMed: 23021004]

7. Jung YO, et al. Nat. Chem. 2013; 5:212-220. [PubMed: 23422563]

8. Schotte F, et al. Proc. Natl. Acad. Sci. USA. 2012; 109:19256-19261. [PubMed: 23132943]

9. Lindenberg AM, et al. Phys. Rev. Lett. 2000; 84:111-114. [PubMed: 11015847]

10. Beaud P, et al. Phys. Rev. Lett. 2009; 103:155702-155706. [PubMed: 19905651]

11. Chapman HN, et al. Nature. 2011; 470:73-77. [PubMed: 21293373] 
12. Holton JM, Frankel KA. Acta Cryst. 2010; D66:393-408.

13. Moffat K. Chem. Rev. 2001; 101:1569-1581. [PubMed: 11709992]

14. Hamm P, Lim M, Hochstrasser RM. J. Chem. Phys. 1998; 102:6123-6138.

15. Porter G. Proc. R. Soc. Lond. A. 1950; 200:284-300.

16. Harwit, M.; Sloane, NJA. Hadamard Transform Optics. Academic Press; 1979.

17. Fernandez F, et al. Anal. Chem. 2002; 74:1611-1617. [PubMed: 12033252]

18. Bolinger L, Leigh J. J. Magn. Reson. 1988; 80:162-167.

19. Garman EF. Acta Cryst. 2010; D66:339-351.

20. Owen RL, Rudino-Pinera E, Garman EF. Proc. Natl. Acad. Sci. USA. 2006; 103:4912-4917. [PubMed: 16549763]

\section{Methods only references}

21. Paithankar KS, Garman EF. Acta Cryst. 2010; D66:381-388.

22. Kabsch WXDS. Acta Cryst. 2010; D66:125-132.

23. Evans PR. Acta Cryst. 2011; D67:282-292.

24. French GS, Wilson KS. Acta. Cryst. 1978; A34:517-525.

25. Murshudov GN, et al. Acta Cryst. 2011; D67:355-367. 

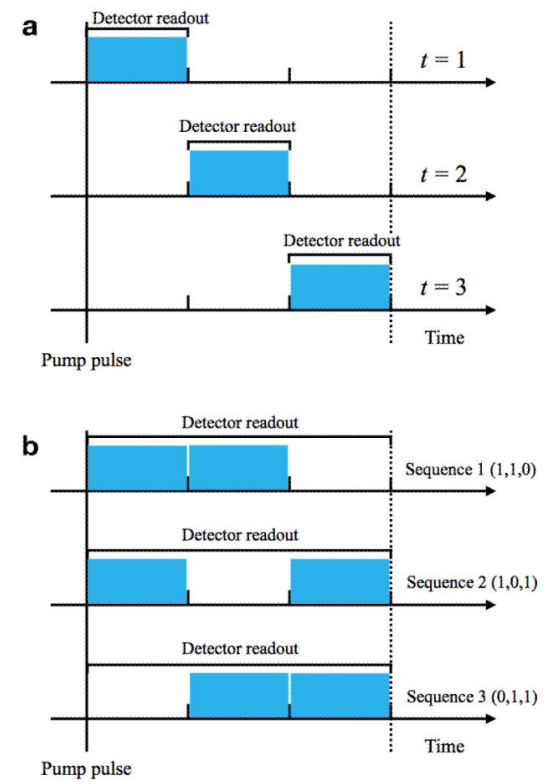

c

$$
\begin{aligned}
{\left[\begin{array}{lll}
1 & 1 & 0 \\
1 & 0 & 1 \\
0 & 1 & 1
\end{array}\right] } & \underset{\mathbf{S}}{\mathbf{W}}
\end{aligned}
$$

Figure 1.

Comparison of classical pump-probe and HATRX methods. (a) The classical pump-probe method, showing three pump-probe time delays. (b) The simplest Hadamard pulse sequence to measure three time points. Note that the detector is read-out only at the end of the whole sequence in this experiment. (c) The $3 \times 3$ Hadamard $\mathbf{S}$ matrix illustrating how each row produces a single summed intensity for each reflection on the detector $\left(w_{1}\right.$, etc.) forming the vector $\boldsymbol{W}$. 


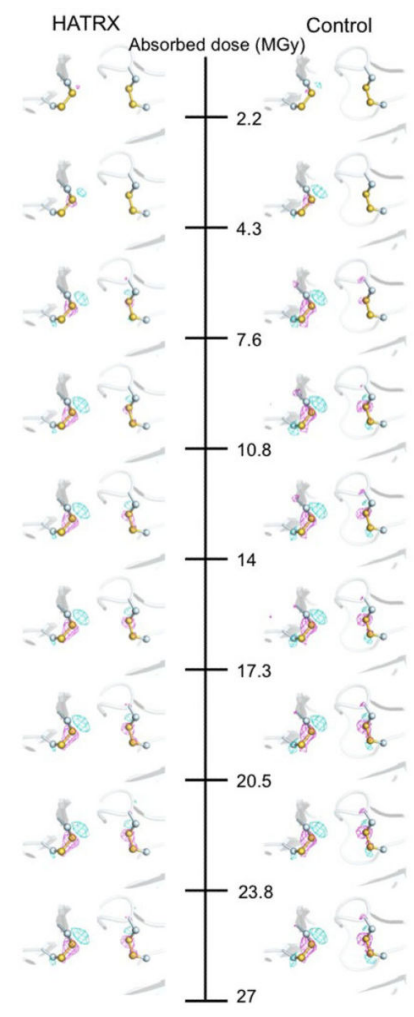

Figure 2.

Difference electron density maps showing the comparison of control and HATRX data. Maps are calculated as $F_{\mathrm{t}}-F_{1}$ (where $t=2,4,7,10,13,16,19,22,25$ ) for the control data (left) and the HATRX data (right). The dose resolution of the experiment is $1.2 \mathrm{MGy}$, which was delivered in $200 \mathrm{~ms}$. The maps are contoured at 3 r.m.s.d. Negative difference density is coloured magenta and positive difference density is coloured cyan. The disulphide bonds between Cys56 - Cys66 and Cys71 - Cys77 are shown. 
a Control data

\begin{tabular}{|c|c|c|c|c|c|c|c|}
\hline Crystal 1 & $1_{1}$ & $22_{1}$ & $3_{1}$ & $\cdots$ & $\cdots$ & $26_{1}$ & $27_{1}$ \\
\hline Crystal 2 & $1_{2}$ & $2_{2}$ & $3_{2}$ & $\cdots$ & $\cdots$ & $26_{2}$ & $27_{2}$ \\
\hline$\vdots$ & $\vdots$ & $\vdots$ & $\vdots$ & & & $\vdots$ & $\vdots$ \\
\hline Crystal 30 & $1_{30}$ & $2_{30}$ & $3_{30}$ & $\cdots$ & $\cdots$ & $26_{30}$ & $27_{30}$ \\
\hline Crystal 31 & $1_{31}$ & $2_{31}$ & $3_{31}$ & $\cdots$ & $\cdots$ & $26_{31}$ & $26_{31}$ \\
\hline
\end{tabular}

b HATRX data for $n=3$

\begin{tabular}{|c|c|c|c|c|c|c|c|c|c|c|c|}
\hline Image number & 1 & 2 & 3 & 5 & 6 & 7 & 9 & $\ldots$ & 25 & 26 & 27 \\
\hline Images to be summed for group 1 & 1 & 1 & 0 & 1 & 0 & 1 & 0 & $\cdots$ & 1 & 1 & 0 \\
\hline Images to be summed for group 2 & 1 & 0 & 1 & 0 & 1 & 1 & 1 & $\cdots$ & 1 & 0 & 1 \\
\hline Images to be summed for group 3 & 0 & 1 & $1 H$ & 1 & 1 & 0 & 1 & $\cdots$ & 0 & 1 & 1 \\
\hline
\end{tabular}

Figure 3.

Schematics showing the experimental design used to demonstrate HATRX. (a) shows how batches were scaled together to generate the control time-resolved datasets. (b) shows how images were summed to create the HATRX images. 1 indicates the image was included in the summed image, 0 that it was not included. 